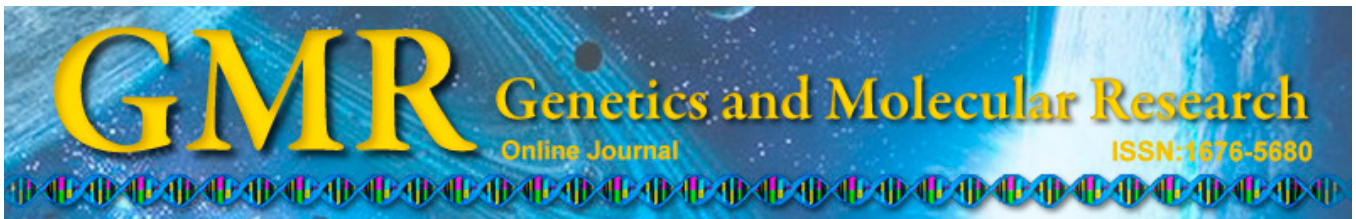

\title{
Clinical significance and levels of blood brain natriuretic peptides in patients with persistent atrial fibrillation before and after catheter ablation
}

\author{
S.Y. Xing, H.L. Wang, P.S. Dong, L.H. Lai, J.H. Zhu, J.F. Zhao and \\ X.Y. Shang \\ Department of Cardiology, \\ The First Affiliated Hospital of Henan Science and Technology University, \\ Luoyang, China \\ Corresponding author: P.S. Dong \\ E-mail: pingshuandong@126.com \\ Genet. Mol. Res. 14 (2): 6953-6959 (2015) \\ Received July 28, 2014 \\ Accepted January 26, 2015 \\ Published June 26, 2015 \\ DOI http://dx.doi.org/10.4238/2015.June.26.3
}

\begin{abstract}
The aim of this study was to observe levels of blood brain natriuretic peptides (BNPs) in patients with persistent atrial fibrillation (AF) before and after catheter ablation. Thirty-six patients with persistent AF (28 successful surgeries and eight recurrent cases) and 36 healthy controls with normal sinus rhythm were recruited for this study. BNP levels in the AF and control groups were measured before and after catheter ablation. BNP levels before surgery were significantly higher in the persistent AF group than in the control group $(\mathrm{P}<0.01)$. The successful surgery group had distinctly lower BNP levels before ablation than the recurrent group $(\mathrm{P}<0.01)$. In the recurrent group, BNP levels $2 \mathrm{~h}$ after ablation were significantly lower than those before ablation $(\mathrm{P}<0.01)$; these levels increased after AF recurrence $(\mathrm{P}$ $<0.01)$ and were comparable with those before ablation $(\mathrm{P}<0.01)$. Logistic regression analysis indicated that the BNP level was an inde-
\end{abstract}


pendent factor for and predictor of AF recurrence $(\mathrm{P}<0.01)$. The BNP level in patients with persistent AF is clinically important in predicting and evaluating AF recurrence after ablation.

Key words: Brain natriuretic peptides; Atrial fibrillation recurrence; Persistent atrial fibrillation; Radiofrequency ablation for atrial fibrillation

\section{INTRODUCTION}

Atrial fibrillation $(\mathrm{AF})$ is the primary risk factor for the most common types of tachyarrhythmia and apoplexy. AF increases mortality rates and disability in patients. Catheter ablation for AF is a novel and rapidly developing treatment strategy that is becoming the focus of treatment for AF. However, AF recurrence after catheter ablation has been challenging. A previous study recently reported that AF patients had increased brain natriuretic peptide (BNP) levels, indicating a correlation with AF (Okçün et al., 2002; Vinch et al., 2004; Tops et al., 2006). Therefore, we observed blood BNP levels in patients with persistent AF and in healthy controls, as well as changes in BNP levels in AF patients before and after catheter ablation, to explore the clinical significance of BNP in the initiation of persistent AF and its recurrence after ablation.

\section{MATERIAL AND METHODS}

\section{General data}

Patients with persistent AF who underwent catheter ablation in our hospital between December 2008 and August 2011 were included in this study. The subjects met the American College of Cardiology, American Heart Association, and European Society of Cardiology's Guidelines for the Management of Patients with AF; namely, patients whose AF symptoms had begun $>7 \mathrm{~d}$ before surgery and who could not recover spontaneously, were included. Thirty-six patients were selected, of which 21 were male and 15 were female, aged $64.3 \pm 7.1$ years, with an AF duration of $15.3 \pm 3.7$ months. The exclusion criteria were as follows: 1) organic heart diseases, such as severe coronary heart disease (CHD), valvular disease, or cardiomyopathy; 2) clinical signs, symptoms, or ultrasonic cardiogram evidence of cardiac insufficiency; 3) severe respiratory disease; 4) pregnancy; 5) thrombus in the left atrium and left auricle; 6) severe chronic disease, such as hypohepatia; 7) recent surgical history and hemorrhagic tendency; 8) thyroid disease; or 9) $>80$ years of age. Additionally, 36 individuals (20 male and 16 female) with a normal sinus rhythm, aged $64.7 \pm 5.3$ years, who were examined in our hospital during the same period and without history of CHD, hypertension, and diabetes, were included as healthy controls in this study. The controls had normal results on physical examination; normal blood glucose levels and blood lipids; normal liver, kidney, and thyroid functions; as well as normal findings on electrocardiogram (ECG); chest radiograph; and color Doppler ultrasound. This study was conducted in accordance with the declaration of Helsinki and with approval from the Ethics Committee of Henan Science and Technology University. Written informed consent was obtained from all participants. 


\section{Operation methods}

The patients were intramuscularly injected low-molecular-weight heparin (LMWH) twice per day, 3 to $5 \mathrm{~d}$ before surgery, and the transesophageal echocardiography was performed to exclude the possibility of thrombus in the left atrium and auricle. Local anesthesia was administered using $1 \%$ lidocaine. The left subclavian vein was punctured, and the coronary sinus electrode was then inserted. The right femoral vein was punctured twice. $\mathrm{L}_{1}$ and $\mathrm{R}_{0}$ Swartz sheaths were used to puncture the interatrial septum, after which heparin was intravenously injected during surgery (carry capacity: $100 \mathrm{U} / \mathrm{kg}$ ) and $1000 \mathrm{U} / \mathrm{h}$ thereafter. Angiography of the left atrium and pulmonary vein was performed, and a circumferential pulmonary electrode was inserted into the pulmonary vein to construct a three-dimensional model of the left atrium and pulmonary vein using an Ensite NavX system (St. Jude Medical, Inc., MN, USA). The temperature-controlled radiofrequency catheter ablation instrument was set to $30 \mathrm{~W}$ and $43^{\circ} \mathrm{C}$. Fentanyl was pumped intravenously and continuously during the surgery. An $8-\mu \mathrm{m}$ irrigated tip ablation catheter was used for electrical isolation along the left and right pulmonary veins until the potentials in both sides of the pulmonary veins were completely isolated, and a bidirectional block was formed during linear ablation. Left atrium linear ablation and local discrete ablation were performed in patients with significant fractionated potentials in the left atrium. We performed ablation in patients with both atrial tachycardia and atrial flutter, and blocked the isthmus between the tricuspid ring and the inferior vena cava (cavotricuspid isthmus) or the mitral valve ring and lower left pulmonary vein (mitral isthmus), according to the activation or entrainment mapping results. Synchronized direct current cardioversion was administered when AF could not be terminated during the surgery.

\section{Follow-up}

After surgery, patients were intramuscularly injected with LMWH for 3 to $5 \mathrm{~d}$. The patients received oral doses of amiodarone and warfarin for three months to maintain an international normalized ratio between 1.8 and 2.5. The administration of antiarrhythmic drugs was terminated three months after catheter ablation. AF recurrence was discovered by body surface ECG or dynamic ECG.

\section{Measurement of BNP}

Venous blood from AF patients was sampled during three separate occasions, namely, on the second day after admission, $2 \mathrm{~h}$ after catheter ablation, and three months after surgery. By contrast, the healthy controls were only sampled once. All patients were asked to fast the night before, and blood was sampled early in the morning. An automatic electrochemical luminescence immunity analyzer Elecsys 2010 (Roche Instrument Center AG, Rotkreuz, Switzerland) and Roche BNP immunoassay kit (Roche Diagnostics GmbH, Mannheim, Germany) were utilized to measure the plasma BNP concentration using double antibody sandwich electrochemiluminescence immunoassay.

\section{Statistical analysis}

Data were statistically analyzed using the SPSS software, version 11.5 (SPSS Inc., IL, 
USA). Measurement data are reported as means \pm standard deviation (SD), and data were compared between groups using the Student $t$-test or ANOVA. Enumerated data were presented as constituent ratios or percentages and analyzed using the $x^{2}$ test. $\mathrm{P}<0.05$ was considered to be statistically significant. Logistic regression was employed to study AF recurrence factors.

\section{RESULTS}

\section{General data}

The differences in age, gender, body mass index (BMI), left ventricular end-diastolic diameter (LVEDD), and left ventricular ejection fraction (LVEF) between the two groups were not statistically significant $(\mathrm{P}>0.05)$. BNP levels in the AF group before ablation was distinctly higher than those in the control group $(\mathrm{P}<0.01$, Table 1$)$.

Table 1. Comparison of general data between AF and control groups ( means \pm SD).
\begin{tabular}{lcccccr}
\hline Groups & Age (years) & Gender (Male/Female) & BMI $\left(\mathrm{kg} / \mathrm{mm}^{2}\right)$ & LVEDD $(\mathrm{mm})$ & LVEF $(\%)$ & BNP $(\mathrm{pg} / \mathrm{ml})$ \\
\hline Control & $64.7 \pm 5.3$ & $20 / 16$ & $24.8 \pm 4.3$ & $49.3 \pm 3.7$ & $64.3 \pm 3.3$ & $46.9 \pm 10.2$ \\
$\mathrm{AF}$ & $64.3 \pm 7.1$ & $21 / 15$ & $25.1 \pm 4.6$ & $50.2 \pm 4.3$ & $62.4 \pm 4.1$ & $135.2 \pm 37.2^{\Delta}$ \\
\hline
\end{tabular}

${ }^{\boldsymbol{\Lambda}}$ Compared with control group, $\mathrm{P}<0.01$

\section{BNP level in AF group}

BNP levels in the successful ablation group significantly decreased $2 \mathrm{~h}$ after surgery $(\mathrm{P}<0.01)$ and declined further after three months $(\mathrm{P}<0.01)$. BNP levels in the successful group before and after ablation were significantly lower than those in the recurrent group ( $\mathrm{P}$ $<0.01$, Table 2).

Table 2. Comparison of BNP levels in successful and recurrent groups before and after catheter ablation (ng/L, means $\pm \mathrm{SD})$.

\begin{tabular}{lcccc}
\hline Groups & Case $(\mathrm{N})$ & Before ablation & Postopetative $2 \mathrm{~h}$ & Posteroperative three months \\
\hline Successful & 28 & $83.1 \pm 38.2$ & $58.7 \pm 25.3^{\mathbf{\Delta}}$ & $49.3 \pm 22.1^{\mathbf{\Delta}}$ \\
Recurrent & 8 & $103.2 \pm 40.3^{\star}$ & $76.3 \pm 31.3^{\mathbf{}}$ & $98.7 \pm 45.3^{\mathbf{}}$ \\
\hline
\end{tabular}

${ }^{\boldsymbol{\Delta}}$ Compared with that before ablation, $\mathrm{P}<0.01 ;{ }^{\wedge}$ Compared with that $2 \mathrm{~h}$ after ablation, $\mathrm{P}<0.01$; ${ }^{\star}$ comparison between groups, $\mathrm{P}<0.01$

\section{BNP level in recurrent group}

BNP levels in the recurrent group significantly decreased $2 \mathrm{~h}$ after ablation $(\mathrm{P}<0.01)$, but increased after $\mathrm{AF}$ recurrence $(\mathrm{P}<0.01)$; this increase was significantly higher than that $2 \mathrm{~h}$ after surgery $(\mathrm{P}<0.01)$, and was comparable with that before ablation. BNP levels in the recurrent group before and after ablation were significantly higher than those in the successful group $(\mathrm{P}<0.01$, Table 2$)$. 


\section{Logistic regression analysis}

Logistic regression analysis was performed with $\mathrm{AF}$ recurrence as the dependent variable and age, gender, BMI, LVEF, LVEDD, and BNP level as the independent variables. The results indicated that BNP level was an independent factor for and predictor of $\mathrm{AF}$ recurrence $(\mathrm{P}<0.01)$.

\section{DISCUSSION}

$\mathrm{AF}$ is a common clinical arrhythmia, and its initiation and maintenance are associated with cardiac electrical remodeling and structural remodeling, but its pathogenesis remains unclear. A recent study (Pourafkari et al., 2014) found significantly increased blood BNP levels in AF patients. BNP is a 32-amino acid polypeptide that is secreted by the ventricle and reflects heart failure severity. BNP has multiple favorable effects, including natriuresis, diuresis, and vasodilation. This polypeptide was first isolated by Sodoh et al. (1988) from brain tissue, and subsequent in vivo and in vitro experiments showed that $60-80 \%$ of plasma BNP is from myocardial cells. Silvet et al. (2013) further confirmed that BNP in AF patients is mainly secreted by the ventricle, which indicates its involvement in the pathogenesis of AF. Moreover, current studies have revealed a close correlation between BNP levels and AF.

We observed serum BNP levels in patients with persistent AF and in healthy controls, as well as changes in BNP levels in AF patients before and after catheter ablation, to explore the clinical significance of BNP in the initiation of persistent AF and its recurrence after ablation. Our study showed that the AF group had distinctly higher BNP levels than the control group before ablation $(\mathrm{P}<0.01)$. These increased levels may result from the overload of heart volume and pressure, increased atrial pressure, and atrial wall tension during AF. BNP is rarely stored in a healthy heart, and its highly productive synthesis is achieved under stimulation. BNP secretion is closely correlated with the volume and pressure load of heart chambers (Beck-da-Silva et al., 2004; Date et al., 2006; Wozakowska-Kaplon and Opolski, 2010; Tang et al., 2011; Barassi et al., 2012; Govindan et al., 2012). In the successful ablation group, BNP levels significantly decreased $2 \mathrm{~h}$ after surgery $(\mathrm{P}<0.01)$ and declined even further after three months $(\mathrm{P}<0.01)$, which is consistent with the results in the literature (Marsiliani et al., 2010; Deftereos et al., 2011; Freynhofer et al., 2011; Higa et al., 2011; Kawabata et al., 2011; Ruggiero et al., 2011; Psychari et al., 2011). After sinus rhythm was restored, the atrium exhibited effective contractions, its auxiliary pump function recovered, hemodynamic rhythm improved, and poor mechanical and volumetric stimulations to atrial cells decreased or disappeared. Moreover, neuroendocrine activation decreased, which restored serum BNP levels. Given the short half-life of BNP (23 min), its level significantly declined $2 \mathrm{~h}$ after AF recovered. This decrease was sustained until three months after the surgery, which indicates that the left atrial structure can undergo reverse remodeling after ablation (Ari et al., 2008; Bakowski et al., 2009; Kallergis et al., 2010; Wozakowska-Kapłon et al., 2010; Wozakowska-Kapłon, 2010). In the recurrent group, BNP levels were significantly higher than that in the successful group before ablation $(\mathrm{P}<0.01)$ and significantly decreased $2 \mathrm{~h}$ after ablation $(\mathrm{P}<0.01)$. However, this level increased after AF recurrence; this increase was significantly higher than that $2 \mathrm{~h}$ after surgery $(\mathrm{P}<0.01)$ and comparable with that before ablation. These results suggest relatively high BNP levels in patients with persistent AF, easy recurrence of AF after radiofrequency ablation, atrial electrical remodeling, and mechanical remodeling after recurrence, enhanced atrial volume and pressure, synthesis and release of BNP by atrial cells, and 
consequent elevated blood BNP level. According to the logistic regression analysis, AF recurrence was a dependent variable, whereas age, gender, BMI, LVEF, LVEDD, and BNP level were independent variables. The results indicated that BNP level was an independent factor for and predictor of AF recurrence $(\mathrm{P}<0.01)$. This finding further clarifies that a loss of atrial rhythmic contraction influences plasma BNP concentrations in patients, and poor mechanical and volumetric burden stimulates BNP secretion. These results provide clinicians with references for the prognosis of $\mathrm{AF}$ and evaluation of recurrence after ablation.

$\mathrm{BNP}$, a circulating hormone secreted by the heart under increased tension, stress, and capacity load of the heart, is clinically important in AF diagnosis as well as in the prediction and evaluation of AF recurrence after ablation. As a biochemical index, BNP detection is convenient, accurate, and repeatable, thus enabling clinicians to screen appropriate subjects for ablation and increase the success rate of surgery.

\section{REFERENCES}

Ari H, Binici S, Ari S, Akkaya M, et al. (2008). The predictive value of plasma brain natriuretic peptide for the recurrence of atrial fibrillation six months after external cardioversion. Turk. Kardiyol. Dern. Ars. 36: 456-460.

Bakowski D, Wozakowska-Kaplon B and Opolski G (2009). The influence of left ventricle diastolic function on natriuretic peptides levels in patients with atrial fibrillation. Pacing. Clin. Electrophysiol. 32: 745-752.

Barassi A, Pezzilli R, Morselli-Labate AM, Lombardi F, et al. (2012). Serum amyloid a and C-reactive protein independently predict the recurrences of atrial fibrillation after cardioversion in patients with preserved left ventricular function. Can. J. Cardiol. 28: 537-541.

Beck-da-Silva L, de Bold A, Fraser M, Williams K, et al. (2004). Brain natriuretic peptide predicts successful cardioversion in patients with atrial fibrillation and maintenance of sinus rhythm. Can. J. Cardiol. 20: 1245-1248.

Date T, Yamane T, Inada K, Matsuo S, et al. (2006). Plasma brain natriuretic peptide concentrations in patients undergoing pulmonary vein isolation. Heart 92: 1623-1627.

Deftereos S, Giannopoulos G, Kossyvakis C, Raisakis K, et al. (2011). Estimation of atrial fibrillation recency of onset and safety of cardioversion using NTproBNP levels in patients with unknown time of onset. Heart 97: 914-917.

Freynhofer MK, Jarai R, Höchtl T, Bruno V, et al. (2011). Predictive value of plasma Nt-proBNP and body mass index for recurrence of atrial fibrillation after cardioversion. Int. J. Cardiol. 149: 257-259.

Govindan M, Borgulya G, Kiotsekoglou A, Saha SK, et al. (2012). Prognostic value of left atrial expansion index and exercise-induced change in atrial natriuretic peptide as long-term predictors of atrial fibrillation recurrence. Europace 14: 1302-1310.

Higa C, Ciambrone G, Donato MS, Gambarte G, et al. (2011). NT-Pro-BNP levels performance before and after reversion to sinus rhythm in patients with preserved ventricular function. Medicina (B. Aires) 71: 146-150.

Kallergis EM, Manios EG, Kanoupakis EM, Mavrakis HE, et al. (2010). Effect of sinus rhythm restoration after electrical cardioversion on apelin and brain natriuretic Peptide prohormone levels in patients with persistent atrial fibrillation. Am. J. Cardiol. 105: 90-94.

Kawabata M, Hirao K, Hachiya H, Higuchi K, et al. (2011). Role of oral amiodarone in patients with atrial fibrillation and congestive heart failure. J. Cardiol. 58: 108-115.

Marsiliani D, Buccelletti F, Carroccia A, Gilardi E, et al. (2010). Natriuretic peptides and atrial fibrillation. Eur. Rev. Med. Pharmacol. Sci. 14: 855-860.

Pourafkari L, Seyedhosseini S, Kazemi B, Esmaili H, et al. (2014). Changes in serum NT-Pro BNP and left atrial BNP levels after percutaneous transvenous mitral commissurotomy in sinus rhythm versus atrial firilation. J. Cardiovasc. Thorac. Res. 6: 175-179.

Okçün B, Yigit Z, Küçükoglu MS, Mutlu H, et al. (2002). Predictors for maintenance of sinus rhythm after cardioversion in patients with nonvalvular atrial fibrillation. Echocardiography 19: 351-357.

Psychari SN, Chatzopoulos D, Iliodromitis EK, Apostolou TS, et al. (2011). C-reactive protein, interleukin 6, and $\mathrm{N}$-terminal pro-brain natriuretic peptide following cardioversion of atrial fibrillation: is there a role of biomarkers in arrhythmia recurrence? Angiology 62: 310-316.

Ruggiero F, Santini L, Gallagher MM, Papavasileiou LP, et al. (2011). Changes in brain natriuretic peptide level as a predictor of AF recurrence after electrical cardioversion. Minerva Cardioangiol. 59: 135-138. 
Silvet H, Hawkins LA and Jacobson AK (2013). Heart rate control in patients with chronic atrial fibrillation and heart failure. Congest. Heart. Fail. 19: 25-28.

Sudoh T, Kangawa K, Minamino N and Matsuo H (1988). A new natriuretic peptide in porcine brain. Nature 332: 78-81.

Tang Y, Yang H and Qiu J (2011). Relationship between brain natriuretic peptide and recurrence of atrial fibrillation after successful electrical cardioversion: a meta-analysis. J. Int. Med. Res. 39: 1618-1624.

Tops LF, Bax JJ, Zeppenfeld K, Jongbloed MR, et al. (2006). Effect of radiofrequency catheter ablation for atrial fibrillation on left atrial cavity size. Am. J. Cardiol. 97: 1220-1222.

Vinch CS, Rashkin J, Logsetty G, Tighe DA, et al. (2004). Brain natriuretic peptide levels fall rapidly after cardioversion of atrial fibrillation to sinus rhythm. Cardiology 102: 188-193.

Wozakowska-Kaplon B (2010). Changes in plasma natriuretic peptide levels in patients with atrial fibrillation after cardioversion. Int. J. Cardiol. 144: 436-437.

Wozakowska-Kaplon B and Opolski G (2010). Exercise-induced natriuretic peptide secretion predicts cardioversion outcome in patients with persistent atrial fibrillation: discordant ANP and B-type natriuretic peptide response to exercise testing. Pacing. Clin. Electrophysiol. 33: 1203-1209.

Wozakowska-Kapłon B, Bartkowiak R, Grabowska U and Janiszewska G (2010). B-type natriuretic peptide level after sinus rhythm restoration in patients with persistent atrial fibrillation - clinical significance. Kardiol. Pol. 68: 781-786. 